\title{
A reliable rabbit model for hyperkinetic pulmonary hypertension
}

\author{
Wei Wang, MD, ${ }^{\mathrm{a}}$ Ruifang Liu, MD, ${ }^{\mathrm{b}}$ Guangqing Cao, MD, ${ }^{\mathrm{b}}$ Fang Zhang, $\mathrm{MD},{ }^{\mathrm{c}}$ Yiqian Zhang, MD, ${ }^{\mathrm{a}}$ \\ Zhongming Zhang, $\mathrm{MD},{ }^{\mathrm{a}}$ and Shuming $\mathrm{Wu}, \mathrm{MD}^{\mathrm{b}}$
}

\begin{abstract}
Objective: To study the mechanisms of vascular remodeling and increased vascular reactivity, a reliable, economic, easy, and stable animal model of hyperkinetic pulmonary hypertension is needed. The purpose of this study was to construct an animal model of hyperkinetic pulmonary arterial hypertension by chronic systemic-pulmonary shunt in young rabbits.

Methods: Thoracotomy was performed through a midsternal incision in 1-month-old rabbits, and anastomosis between the left carotid artery and pulmonary artery trunk created a chronic left-to-right shunt. After 3 months, the shunted artery was checked by echocardiography. Systolic, diastolic, and mean pulmonary arterial pressures were measured by microcatheterization. The pathologic changes of small pulmonary arteries were observed after staining with hematoxylin and eosin. Thickness and area indices were calculated.
\end{abstract}

Results: High-flow pulmonary hypertension was successfully established in 24 rabbits 3 months after operation. Relative to a sham operation group, the systolic, diastolic, and mean pulmonary arterial pressures were obviously increased in the experimental group $(P<.05)$. Histologic examination showed that the thickness of arterial wall increased, the lumen became narrowed, and thickness and area indices increased in small pulmonary arteries $(P<.05)$.

Conclusions: We constructed a model mimicking the aberrant hemodynamic state in children with congenital heart disease with increased pulmonary blood flow to produce early characteristic morphology of hyperkinetic pulmonary hypertension. This method may provide an economic, easy, and stable animal model to study the mechanisms of pulmonary vascular remodeling in hyperkinetic pulmonary hypertension. (J Thorac Cardiovasc Surg 2010;140:395-9)

Hyperkinetic pulmonary arterial hypertension, a common complication of congenital heart disease with increased pulmonary blood flow, seriously affects prognosis and increases morbidity and mortality associated with repair operations. ${ }^{1}$ The presence of a systemic-pulmonary communication results in increased pulmonary blood flow as pulmonary vascular resistance normally decreases after birth. Progressive structural and functional abnormalities of the pulmonary vascular bed eventually occur if the communication is not closed. Finally, severe pulmonary hypertension results in right-to-left shunt with secondary severe cyanosis and heart failure. Early structural changes are reversible after surgical correction, but late severe structural changes are

\footnotetext{
From the Department of Cardiothoracic Surgery, ${ }^{\text {a }}$ Affiliated Hospital of Xuzhou Medical College, Xuzhou, China; the Department of Cardiovascular Surgery, ${ }^{\mathrm{b}}$ Qilu Hospital, Shandong University, Jinan, China; and the Department of Rheumatology, Second Affiliated Hospital of Xuzhou Medical College, Xuzhou, China.

Supported by grants No. 30571845 of the National Natural Science Foundation of China, No. BK2007033 of the Natural Science Foundation of Jiangsu Province of China, and 07KJD310218 of the Education Department of Jiangsu province of China.

Disclosures: None.

W.W. and R.L. contributed equally to this work.

Received for publication Jan 8, 2009; revisions received March 30, 2009; accepted for publication April 20, 2009; available ahead of print June 7, 2010.

Address for reprints: Shuming Wu, MD, PhD, Department of Cardiovascular Surgery, Qilu Hospital of Shandong University, Jinan, China (E-mail: shumingwumd@126. com).

$0022-5223 / \$ 36.00$

Copyright (c) 2010 by The American Association for Thoracic Surgery

doi:10.1016/j.jtcvs.2009.04.071
}

irreversible and progressive. The status of the pulmonary vasculature is thus the principal determinant of the clinical course and surgical treatment. ${ }^{2}$

The mechanisms of vascular remodeling remain incompletely understood, although the vascular morphology of pulmonary hypertension has been well described. To study this disease, a reliable animal model of hyperkinetic pulmonary hypertension is necessary. At present there are many difficulties and high technical requirements impeding establishment of an animal model of hyperkinetic pulmonary arterial hypertension through surgical operations. ${ }^{3-5}$ It would be valuable to launch an animal model that would be economic, relatively easy, stable, and closer to simulating the pathophysiologic status of human congenital heart disease. Anastomosis of the left carotid artery to the main pulmonary artery in young rabbits was performed in our animal laboratory to observe the hemodynamic pathophysiologic status and the morphology of pulmonary vascular bed in an attempt to provide an easily established, reliable, and economic small animal model of hyperkinetic pulmonary hypertension.

\section{MATERIALS AND METHODS \\ Experimental Animals}

The study protocol was approved by the animal care committee at the Xuzhou Medical College of China. The animals received humane care in compliance with the Guide for the Care and Use of Laboratory Animals of Jiangsu Province of China. 


\section{Abbreviations and Acronyms \\ DPAP $=$ diastolic pulmonary arterial pressure \\ MPAP $=$ mean pulmonary arterial pressure \\ $\mathrm{SPAP}=$ systolic pulmonary arterial pressure}

Sixty 1-month-old New Zealand albino rabbits (including male and female animals) with a body weight of $540 \pm 24 \mathrm{~g}$ were obtained from the Experimental Animal Center of Xuzhou Medical College (Xuzhou, China). The animals were randomly divided into 2 groups: the experimental group (shunt group) consisted of 40 rabbits that underwent left carotid artery-pulmonary arterial shunt, whereas the control group consisted of 20 rabbits that underwent thoracotomy alone (sham operation).

\section{Surgical Protocol}

The animals were kept fasting for 4 hours before the operation and were medicated in advance with intramuscular injections of ketamine $(2 \mathrm{mg} / \mathrm{kg})$ and midazolam $(1 \mathrm{mg} / \mathrm{kg}$ ), followed by an intravenous bolus of ketamine $(1 \mathrm{mg} / \mathrm{kg})$ administered through the ear vein. Stable anesthesia was maintained with a continuous intravenous infusion of fentanyl $(30 \mu \mathrm{g} /[\mathrm{kg} \cdot \mathrm{h}])$ and midazolam $(2 \mathrm{mg} /[\mathrm{kg} \cdot \mathrm{h}])$.

Shunt group. The rabbit was immobilized in a supine position. Electrocardiographic leads were inserted into the limbs for monitoring. The mandible was pulled down and forward, and the tongue was pulled out to keep breathing smooth. Oxygen was supplied by face mask with an inspired oxygen fraction of 0.4. A median sternotomy was performed after standard skin preparation. The pericardium was opened along the main pulmonary and suspended with tacking sutures. The main pulmonary artery was dissected from the aorta, and the skin incision was extended to the neck to expose the left carotid artery. Heparin (20 units) was injected intravenously, and papaverine $(1 \%)$ was dripped onto the surface of arteries for dilation. The left carotid artery was cut at 1.2 to $1.5 \mathrm{~cm}$ from its origin. The distal end was ligated, and the proximal end was clamped with a bulldog clamp. The main pulmonary artery was clamped with a mini side-biting vascular clamp, and a 4-mm longitudinal incision was made. Then the proximal end of left carotid artery was trimmed into a $45^{\circ}$ bevel and anastomosed to the pulmonary artery trunk with continuous 7-0 Proline sutures (Ethicon Inc, Somerville, NJ). The mini side-biting vascular clamp was gradually released before the suture was tied, allowing air to escape through the suture line (Figure 1). A thrill could be palpated if the shunt vessel was patent. The rabbit was returned to the cage, where it had free access to food and water once it awoke. Penicillin G and procaine ( 0.5 million units) were administered intravenously during surgery and daily thereafter for 2 days after operation. Aspirin $(2 \mathrm{mg} /[\mathrm{kg} \cdot \mathrm{d}])$ was administered orally for anticoagulation on the second postoperative day.

Control group. The control rabbits underwent thoracotomy alone with the same anesthesia.

\section{Echocardiographic and Color Doppler Studies}

Transthoracic 2-dimensional, M-mode, and color Doppler imaging were performed in all animals with an ultrasonographic system (Sonos 5500; Koninklijke Philips Electronics N.V., Eindhoven, The Netherlands) at 2 weeks, 1 month, and 3 months after operation. The animals were anesthetized with injection of ketamine $(100 \mathrm{mg} / \mathrm{kg}$ intraperitoneally) and xylazine $(7.5 \mathrm{mg} / \mathrm{kg}$ intraperitoneally), and the skin of the chest was shaved to measure the blood flow of the shunt vessel by transthoracic echocardiography.

\section{Hemodynamic Study}

Measurements were done 3 months later, with the same anesthetic regimen as for the first procedure. Anesthesia was maintained until measurements were complete. The anaesthetized rabbit was immobilized in the supine position. Electrocardiographic leads were inserted into limbs. The thorax was entered through the previous incision. The shunt artery was freed and cycled with 4-0 silk sutures. A purse-string suture was made on the trunk of pulmonary artery distal to the anastomosis. A polyvinyl catheter was inserted into the pulmonary artery, and the hemodynamic variables were monitored continuously by Maclab/8s (M8680; ADInstruments Pty Ltd, Bella Vista, NSW, Australia). Systolic pulmonary arterial pressure (SPAP), diastolic pulmonary arterial pressure (DPAP), and mean pulmonary arterial pressure (MPAP) were recorded before and after the shunt vessel was occluded. With the same procedure, a purse-string suture was made on the aorta and a catheter was inserted to measure and record the systemic arterial pressure.

\section{Index of Right Ventricular Hypertrophy}

Each animal was killed with an overdose of barbital, and the heart-lung block was immediately harvested. The right ventricle was dissected from the left ventricle and septum and weighed separately. The right ventricular weight index, a measurement of right ventricular hypertrophy, was expressed as a ratio of the right ventricular weight to the combined weights of the left ventricle and septum. ${ }^{6}$

\section{Pathologic Study of the Lungs}

The lungs were rinsed with $4^{\circ} \mathrm{C}$ saline solution and phosphate-buffered saline-solution immediately after harvesting. Tissues from each lobe of the lung were removed and fixed with $4{ }^{\circ} \mathrm{C} 4 \%$ paraformaldehyde for 4 hours, dehydrated through graded alcohols, and embedded in paraffin. Sections $(5 \mu \mathrm{m})$ were cut from each block and stained with hematoxylin and eosin for routine light microscopic examination. In each slide, 10 small muscular pulmonary arteries of variable diameter $(80-200 \mu \mathrm{m})$ were studied. The medial thickness and the area of the lumen were measured (5 transects of pulmonary artery for each slice selected randomly) with the QWIN100 imaging analyzer system (Leica Microsystems GmbH, Wetzler, Germany), and the thickness index ([1-inner diameter of vessel]/outer diameter of vessel) and the area index ([1-lumen area $] /$ total area of vessel) were calculated. ${ }^{7}$

\section{Statistical Analysis}

The baseline hemodynamic variables, systemic arterial blood gas values, and $\mathrm{pH}$ were calculated and expressed as mean $\pm \mathrm{SD}$. The variables of the rabbits with shunts were compared with those of the control rabbits by unpaired $t$ test. The variables of the rabbits with shunt before shunt closure were compared with those after shunt closure and with those of control rabbits by repeated-measures analysis of variance.

\section{RESULTS \\ Survivals}

The operations in the shunt group took 1.5 to 2 hours. After the shunt operation, slight shock and an audible continuous murmur were maintained on the left side of the chest. Rabbits with shunts had higher resting respiratory rates and were less active than control rabbits. There were no differences in weight and fur between the groups.

Six rabbits died during the operation: 2 died of breath failure related to pneumothorax because the pleura was broken, and another 4 died of injury to the pulmonary artery and bleeding during vessel anastomosis. Success rate of anastomosis was $85 \%$. Seven animals died after the operation: 2 died of cardiac tamponade, 3 died of infection in the lung and incision, and 2 died of unknown causes (probably of heart failure). Twenty-seven rabbits $(67 \%)$ remained alive 


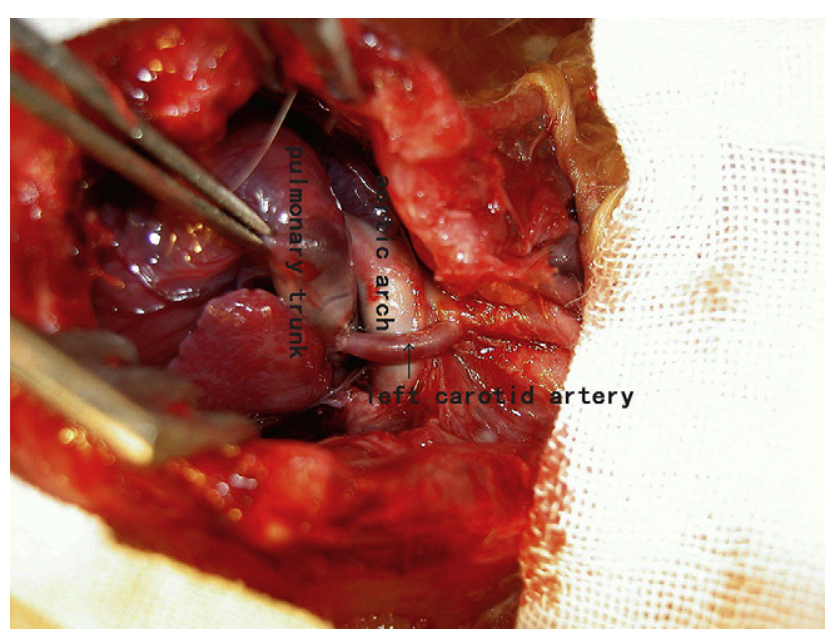

FIGURE 1. Illustration of surgical technique. Anastomosis between left carotid artery and pulmonary trunk.

at the end of the study ( 3 months), but 3 rabbits had to be excluded from analysis because of unsuccessful shunt surgery. In the control group (sham operation, $n=20$ ), 1 died of anesthesia during operation, 2 died of pneumothorax, and 3 died after the operation. Fourteen control rabbits survived at the end of the study (3 months), for a survival of $70 \%$.

\section{Echocardiographic and Color Doppler Studies}

An abnormal vessel connecting the aorta and pulmonary artery could be found in rabbits that had undergone successful shunting. Colorful blood flow could be seen running through the shunt vessel into the pulmonary artery during the whole cardiac cycle (Figure 2). A continuous waveform could be obtained with continuous-wave Doppler at the shunt vessel. The maximum velocity of shunt flow jet was 2.8 to $4.4 \mathrm{~m} / \mathrm{s}$, average $3.23 \pm 0.62 \mathrm{~m} / \mathrm{s}$, and the average differential of flow pressure between aorta and the pulmonary artery was $54 \pm 6 \mathrm{~mm} \mathrm{Hg}$. Among 27 surviving rabbits in the shunt group, patent shunts were found in 24 and the shunt vessel was blocked in 3 within 2 weeks after the operation.

\section{Hemodynamic Study}

Compared with the sham operation (control) group, SPAP, DPAP, and MPAP before the shunt vessel closure were all significantly increased in the shunt group $(P=.0001)$. After closure of the shunt vessel, SPAP, DPAP, and MPAP were decreased $(P=.0374, P=.0423$, and $P=.0392$, respectively) in the shunt group but still higher than corresponding values in the sham operation group ( $P=.0054, P=.0143$, and $P=.0174$, respectively). Relative to the sham operation group, the shunt group showed no remarkable change in average systolic blood pressure (Table 1).

\section{Index of Thickness of Right Ventricle and Change in Blood Oxygenation}

In the shunt group, the ratio of right ventricular weight to the left ventricular and septum weights combined, the index of right ventricular hypertrophy, was significantly increased 3 months after the shunt operation relative to the sham operation group $(P=.0001)$. Relative to the sham operation group, an obvious decrease in arterial $\mathrm{Po}_{2}$ was found in the shunt group $(P=.0124$; Table 2$)$.

\section{Pathologic Examination of the Lung}

Muscular pulmonary arteries showed medial hypertrophy of 80 to $200 \mu \mathrm{m}$ in the shunt group. The thickness and area indices in shunt group were significantly higher than corresponding values in the sham operation group $(0.64 \pm 0.13 \mathrm{vs}$ $0.31 \pm 0.11$ and $0.82 \pm 0.13$ vs $0.42 \pm 0.12$, respectively; Figure 3).

\section{DISCUSSION}

Currently, the animal models most frequently used to study the pathophysiology of pulmonary arterial hypertension involve hypoxia- and monocrotaline-induced pulmonary arterial hypertension. ${ }^{8,9}$ The shunted pulmonary hypertension model has rarely been reported because of technical problems and high mortality. Drug- or low
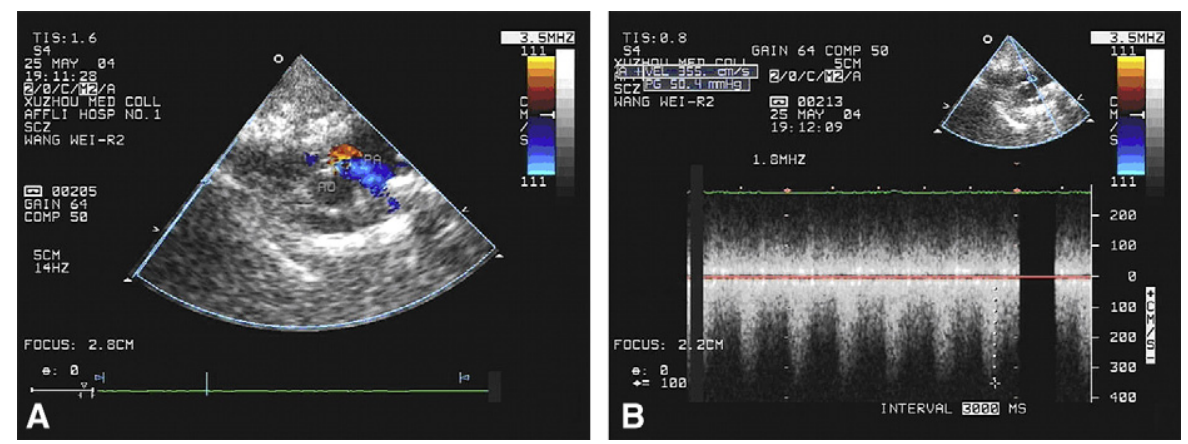

FIGURE 2. Three months after operation, shunted artery was detectable by echocardiography. A, Colorful blood flow can be seen running through shunt vessel into pulmonary artery during entire cardiac cycle. B, Continuous waveform can be detected in shunt vessel with continuous-wave Doppler. Maximum velocity of shunt flow jet was $2.8 \pm 4.4 \mathrm{~m} / \mathrm{s}$ (average $3.23 \pm 0.62 \mathrm{~m} / \mathrm{s}$ ), and average differential of flow pressure between aorta $(A O)$ and pulmonary artery $(P A)$ was $54 \pm 6 \mathrm{~mm} \mathrm{Hg}$. 
TABLE 1. Change of hemodynamic status 3 months after operation $(\mathbf{n}=38)$

\begin{tabular}{lccc}
\hline & & \multicolumn{2}{c}{ Shunt $(\mathbf{n}=\mathbf{2 4})$} \\
\cline { 3 - 4 } & Sham $(\mathbf{n}=\mathbf{1 4})$ & Before closure & After closure \\
\hline Pulmonary arterial pressure & & & \\
$\quad$ Systolic & $14.6 \pm 3.4$ & $28.4 \pm 5.7^{*} \dagger$ & $24.7 \pm 4.6^{*}$ \\
Diastolic & $8.2 \pm 2.8$ & $14.3 \pm 3.3^{*} \dagger$ & $12.6 \pm 3.0^{*}$ \\
$\quad$ Mean & $11.2 \pm 3.2$ & $19.2 \pm 3.7^{*} \dagger$ & $16.6 \pm 3.4^{*}$ \\
Mean systemic & $67.2 \pm 11.1$ & $69.3 \pm 12.4$ & $71.4 \pm 11.6$ \\
$\quad$ & & & \\
arterial pressure & &
\end{tabular}

All data are mean $\pm \mathrm{SD}$ in millimeters of mercury. $* P<.05$ versus sham operation group. $\dagger P<.05$ versus after closure of shunt

oxygen-induced pulmonary arterial hypertension, however, is different in pathophysiology from the hyperkinetic pulmonary arterial hypertension caused by congenital heart disease. It is therefore inaccurate to study pulmonary arterial hypertension secondary to congenital heart disease with models of hypoxia- and monocrotaline-induced pulmonary arterial hypertension. ${ }^{10}$

In this study, 1-month-old rabbits were used to establish chronic systemic-pulmonary shunting by anastomosis of the left carotid artery to the pulmonary arterial trunk, to simulate the pathologic physiologic status of left-to-right shunt congenital heart disease. These young rabbits had good tolerance to the shunt operation. Most casualties were from operative complications, such as infection and hemorrhage, with few dying of heart failure. Three months after the operation, relative to the sham operation group the shunt group had remarkable increases in pulmonary arterial pressure and right ventricle hypertrophy. In reference to the hemodynamic change of left-to-right shunt congenital heart disease, it is defined the standard of pulmonary arterial hypertension that SPAP is greater than $30 \mathrm{~mm} \mathrm{Hg}$ or the ratio of SPAP to systolic systemic arterial pressure is greater than $20 \%$ with occurrence of pathologic change. ${ }^{11}$ In this study, all of the rabbits with successful vessel anastomosis in the shunt group met this standard.
TABLE 2. Index of thickness of right ventricle and arterial $\mathrm{Po}_{2}$ $(\mathbf{n}=\mathbf{3 8})$

\begin{tabular}{|c|c|c|}
\hline & Sham $(n=14)$ & Shunt $(n=24)$ \\
\hline Arterial $\mathrm{Po}_{2}(\mathrm{~mm} \mathrm{Hg})$ & $96.4 \pm 3.3$ & $84.4 \pm 4.1^{*}$ \\
\hline $\begin{array}{l}\text { Right ventricular } \\
\text { thickness index }\end{array}$ & $0.25 \pm 0.02$ & $0.32 \pm 0.03^{*}$ \\
\hline
\end{tabular}

The key to establishing an animal model of left-to-right shunt heart disease is control of the quantity of shunt blood. Rondelet and others ${ }^{12}$ adopted the operation of prosthetic vessel bypass shunt between the aorta and the pulmonary artery, which can form pulmonary arterial hypertension 2 months later, but their study showed that the quantity of shunted blood is difficult to control because a small shunt seldom forms pulmonary arterial hypertension and large shunt often causes death. The hemodynamic status is unstable, and the prosthetic vessel has a higher obstruction rate because of distortion and thrombosis of the graft vessel. ${ }^{12}$ The left carotid artery of the rabbit has sufficient length to create systemic to pulmonary shunt and form moderate shunt flow with end-to-side anastomoses to the pulmonary artery because of its appropriate diameter. Furthermore, the artery can grow to reduce the differentials in individuals. In our study, to prevent vascular spasm, papaverine was dropped to the surface of the arteries to obtain a large enough diameter of the anastomosis. Aspirin was orally administered 24 hours after the operation to reduce the rate of thrombosis. The 3-month shunt patency rate in our model was $89 \%$, which was much higher than that in the prosthetic vessel bridge model. In comparison with the side-to-side anastomosis of the aorta and pulmonary artery, with this method it is easy to control the size of the anastomosis to prevent acute heart failure because of an overly large diameter of shunt and either obstruction of the shunt or inability to form pulmonary hypertension because of an overly small diameter of shunt.
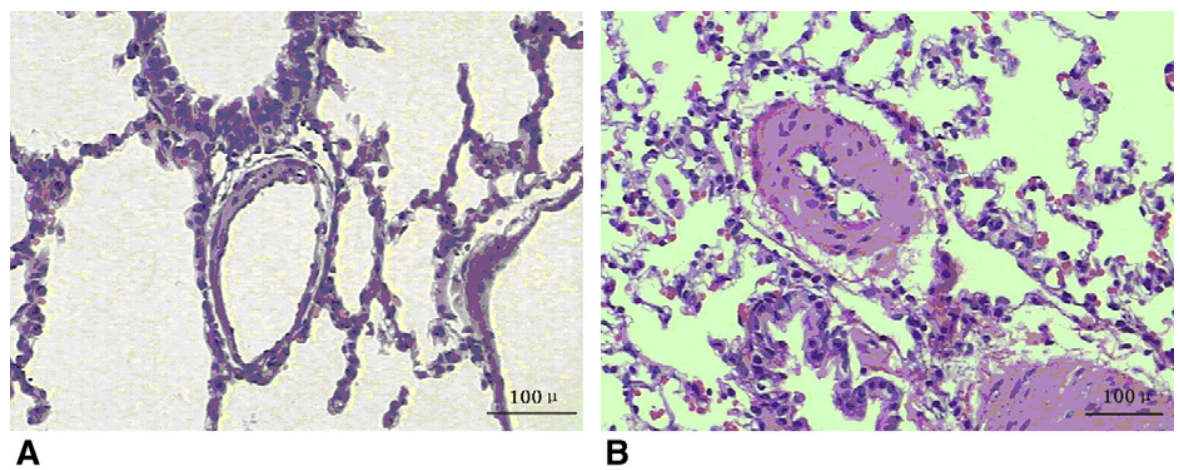

FIGURE 3. Pathologic changes in pulmonary artery 3 months after operation (hematoxylin and eosin stain, $\times 200$ ). A, Sham operation group. B, In shunt group, thickness of arterial wall increased and lumen became narrowed. 
Relative to the control group, SPAP, DPAP, and MPAP in the shunt group were obviously increased 3 months after operation; pulmonary arterial pressures were partially decreased after closure of shunt vessel but remained higher than those in the control group because of the pathologic change in pulmonary vessel structure.

In summary, an animal model of postnatal pulmonary hypertension could be established by anastomosis of the left carotid artery to the pulmonary arterial trunk in young rabbits. The model closely represents the aberrant hemodynamic state in children with congenital heart disease with increased pulmonary blood flow and also produces early and characteristic morphologic changes in the pulmonary vasculature. This method could provide an economic, easy, and stable animal model to study the mechanisms of pulmonary vascular remodeling in kinetic pulmonary hypertension.

\section{References}

1. Roos-Hesselink JW, Meijboom FJ, Spitaels SE, Van Domburg R, Van Rijen EH, Utens EM, et al. Outcome of patients after surgical closure of ventricular septal defect at young age: longitudinal follow-up of 22-34 years. Eur Heart J. 2004; 25:1057-62.
2. Hoffman JI, Rudolph AM, Heymann MA. Pulmonary vascular disease with congenital heart lesions: pathologic features and causes. Circulation. 1981;64:873-7.

3. Junbao D, Hui Y, Bing W, Jian L, Jianguang Q, Chaoshu T, et al. Effect of L-arginine on collagen of high flow-induced pulmonary arterial remodeling. Circ J. 2005;69:603-8.

4. Chu D, Sullivan CC, Du L, Cho AJ, Kido M, Wolf PL, et al. A new animal model for pulmonary hypertension based on the overexpression of a single gene, angiopoietin-1. Ann Thorac Surg. 2004;77:449-56.

5. Rabinovitch M. The mouse through the looking glass: a new door into the pathophysiology of pulmonary hypertension. Circ Res. 2004;94:1001-4.

6. Reddy VM, Meyrick B, Wong J, Khoor A, Liddicoat JR, Hanley FL, et al. In utero placement of aortopulmonary shunts: a model of postnatal pulmonary hypertension with increased pulmonary blood flow in lambs. Circulation. 1995;92:606-13.

7. Nagumo K, Yamaki S, Takahashi T. Extremely thickened media of small pulmonary arteries in fatal pulmonary hypertension with congenital heart disease-a morphometric and clinicopathological study. Jpn Circ J. 2000;64: 909-14.

8. Ambalavanan N, Bulger A, Murphy-Ullrich J, Oparil S, Chen YF. Endothelin-A receptor blockade prevents and partially reverses neonatal hypoxic pulmonary vascular remodeling. Pediatr Res. 2005;57:631-6.

9. Mathew R, Huang J, Shah M, Patel K, Gewitz M, Sehgal PB. Disruption of endothelial-cell caveolin- $1 \alpha / \mathrm{raft}$ scaffolding during development of monocrotaline-induced pulmonary hypertension. Circulation. 2004;110:1499-506

10. Ullmann MV, Gorenflo M. Bosentan and overcirculation-induced experimental pulmonary arterial hypertension [letter]. Circulation. 2003;108:e157.

11. Haworth SG. Pulmonary hypertension in the young. Heart. 2002;88:658-64.

12. Rondelet B, Kerbaul F, Van Beneden R, Motte S, Fesler P, Hubloue I, et al. Signaling molecules in overcirculation-induced pulmonary hypertension in piglets: effects of sildenafil therapy. Circulation. 2004;110:2220-5. 